\title{
Waveguiding at 1550nm using Photonic Crystal Structures in Silicon on Insulator Wafers
}

\author{
Dusan Nedeljkovic \\ Centre Européen de Recherche de Fontainebleau, Corning, SA 77210 Avon, France \\ nedelikod@corning.com \\ Marko Loncar \\ Department of Electrical Engineering, Caltech, Pasadena, California 91125 USA \\ Sergey Kuchinsky \\ Corning Scientific Center, Corning, Ltd. 199034, St. Petersburg, Russia \\ Mikhail Mikhailov \\ Corning Scientific Center,Corning, Ltd. 199034, St. Petersburg, Russia \\ Axel Scherer \\ Department of Electrical Engineering, Caltech, Pasadena, California 91125 USA \\ Thomas P. Pearsall \\ Centre Européen de Recherche de Fontainebleau, Corning, SA 77210 Avon, France
}

\begin{abstract}
Design, fabrication and light guiding in planar photonic crystal structures including sharp corners with a bending radius less than 1 micron are demonstrated at $1550 \mathrm{~nm}$ wavelength in silicon waveguides on silicon dioxide substrates. Photon confinement in the sample plane was achieved by a photonic crystal structure while confinement in the vertical direction was achieved by index of refraction contrast.

(C)2000 Optical Society of America

OCIS codes: (050.0050) Diffraction and gratings; (130.0130) Integrated optics
\end{abstract}

\section{Introduction}

Planar photonic crystal waveguides and devices may offer the possibility to build photonic circuits with greater density and new functionality compared to existing waveguide devices that are based on the control of light by refraction. The primary vehicles for this research are the study of microcavity lasers as a path to understanding new functionality, and the propagation of light around sharp bends as the path to increasing the packing density of planar optical circuits [1-6]. In this paper we report our results on the successful demonstration of coupling and guiding of light in a planar photonic circuit incorporating sharp bends.

\section{Theoretical Analysis}

Waveguide structures analyzed here utilize an optically thin, high-index membrane to confine light in the vertical direction (by way of Total Internal Reflection) and a two-dimensional Photonic Crystal structure to confine light in the lateral direction. Three-dimensional Plane-Wave-Expansion Frequency Domain analysis was performed in order to calculate band diagram of photonic crystal, as well as to find the dispersion diagram of guided modes in case of the waveguide. The optimal period of a square lattice of holes was found to be $a=510 \mathrm{~nm}$ and the ratio of the hole diameter to period, $r / a=0.4$. Thickness of the slab was $d=0.6 a$. Parameters for the hexagonal lattice can be found elsewhere [1]. 


\section{Sample Preparation}

Photonic crystal structures were designed to confine light in a silicon waveguide at $1550 \mathrm{~nm}$, by drilling air holes at periodic intervals around the waveguide region. Both square and hexagonal structures have been studied. The pattern design was transferred to a silicon-on-insulator wafer using direct-write e-beam lithography with $20 \mathrm{~nm}$ resolution. The pattern was etched into the silicon layer by chemically assisted ion beam etching (CAIBE) or reactive ion etching in chlorine containing plasma. A SEM micrograph showing a typical structure is presented in Figure 1.

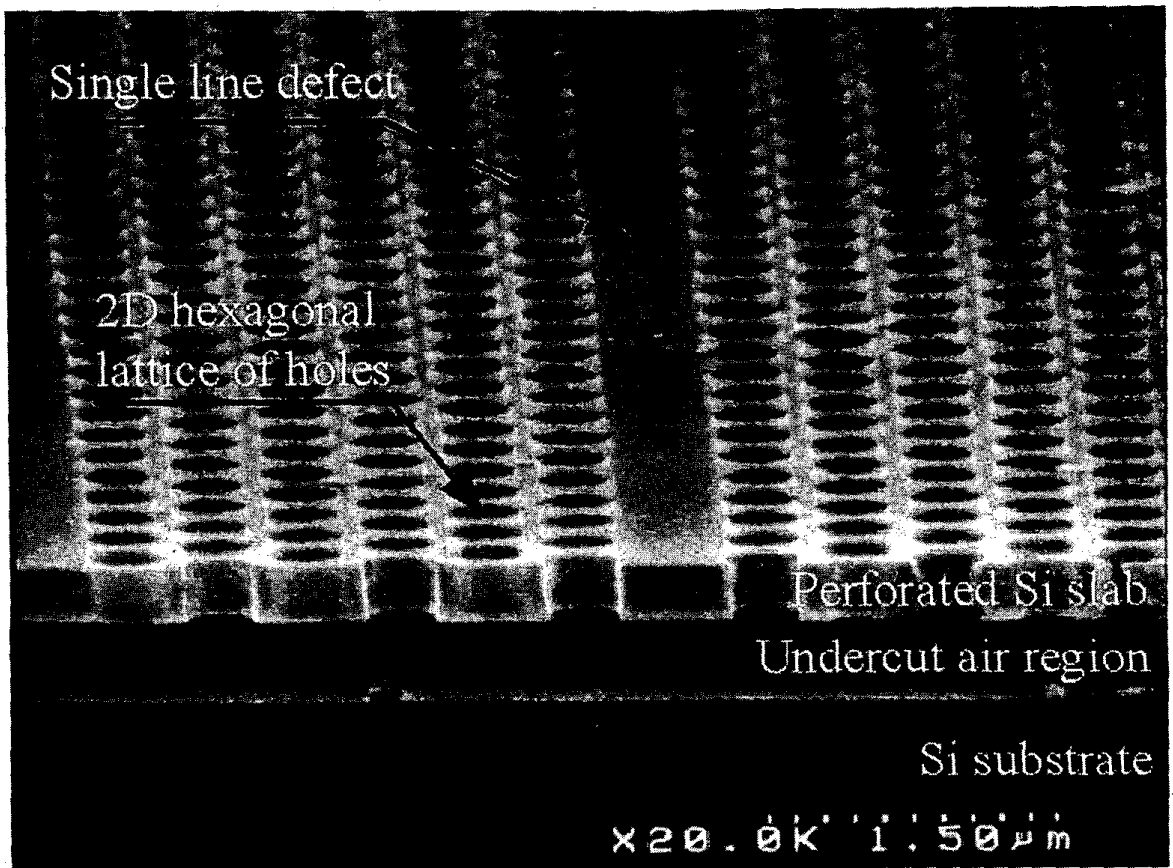

Fig. 1. Scanning electron micrograph showing the photonic crystal confinement regions surrounding a central waveguide in silicon. Light of $1500 \mathrm{~nm}$ wavelength is confined in the plane by the photonic crystal period. Vertical confinement is achieved by the difference in refractive index

Following processing the samples were characterized to confirm the structure. Some samples received an additional $\mathrm{HF}$ etch to remove $\mathrm{SiO}_{2}$ layer underneath the waveguide in order to form free-standing membrane as a way of further improving the refractive confinement perpendicular to the plane of waveguiding.

\section{Optical Characterization}

A tunable semiconductor diode laser $(4 \mathrm{~mW})$ was used to characterize optical transmission. Butt-coupling of a single-mode fiber was used to introduce the laser output into the photonic crystal. Only light of TE polarization could be coupled into the structure, consistent with expected photonic crystal confinement properties. Waveguiding performance can be observed by visualization of the guiding structure with an infrared television camera. The example of light propagation around a $60^{\circ}$ bend is shown in Fig. 2. Simultaneous imaging of the photonic crystal shows clearly that the light is confined to the $\mathrm{Si}$ waveguide in the center of the structure. 


\section{TuC6-3}

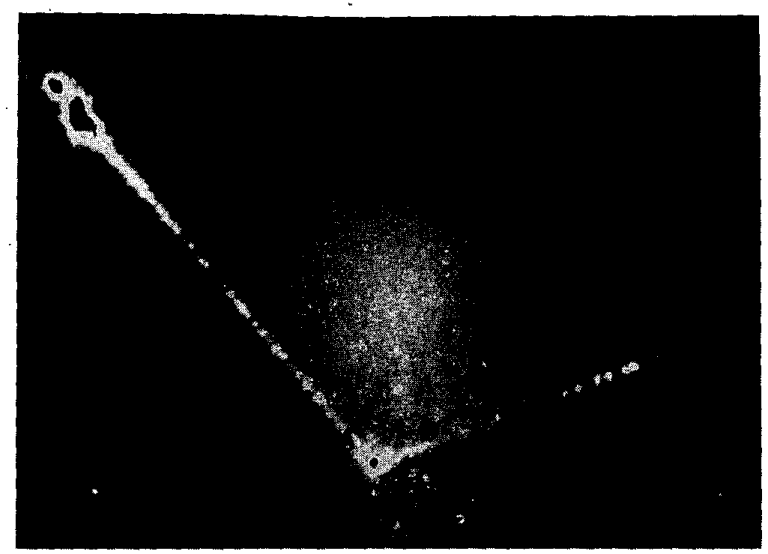

Fig. 2. Guiding of light around a $60^{\circ}$ bend in a photonic crystal waveguide ,

We have used coupling into leaky modes to visualize light propagation through the photonic crystal guides. When the coupling to guided modes is optimized, no light is scattered out the top surface, and a well-defined mode is seen to exit the guide. Under these conditions the loss is below the sensitivity of our measurement which is $8 \mathrm{~dB} / \mathrm{cm}$. The measured performance appears to represent significant progress relative to previous efforts to control the flow of $1550 \mathrm{~nm}$ light by planar photonic crystal waveguides. Because the loss is so low, it has not yet been possible to determine the role of factors such as surface roughness, fabrication errors to the measured performance.

\section{Conclusions}

Photonic crystal waveguides have been designed and fabricated for operation at 1550nm. Experimental measurements show that $1550 \mathrm{~nm}$ light can be guided in these structures, with properties that are consistent with photonic crystal confinement. While on one hand our research results represent encouraging progress toward the use of photonic crystal structures in planar photonic circuits, on the other they show that guiding conditions for real guides in three dimensions are more restrictive than previously thought using two-dimensional modeling. We have measured unambiguous guiding in these structures over the entire length of the sample. Under appropriate coupling conditions, this transmission appears to have tolerably low loss, a feature that is currently under study in our laboratory.

\section{References}

1. M. Loncar, D. Nedeljkovic, T. Doll, J. Vuckovic, A. Scherer, and T.P. Pearsall, "Waveguiding in Planar Photonic Crystals", Appl. Phys. Lett. 77, 1937-39 (2000).

2. O. Painter et al., "Room Temperature Photonic Crystal Defect Lasers at Near Infrared Wavelengths in InGaAsP", IEEE J. of Lightwave Technology, 17, no. 11, pp. 100-107, Nov. 1999.

3. A. Mekis et al, "High Transmission through Sharp Bends in Photonic Crystal Waveguides", Phys. Rev. Letters, 77, no. 18, pp. 3787-3790, Oct. 1996.

4. T. Baba et al., "Observation of Light Propagation in Photonic Crystal Optical Waveguides With Bends", Electronic Letters, 35, no. 8, pp. 654-655, Apr. 1999.

5. Shawn-Yu Lin et al., "Experimental Demonstration of Guiding and Bending of Electromagnetic Waves in a Photonic Crystal", Science, 282, pp. 274-276, Oct. 1998.

6. J. S. Foresi et al., "Photonic-Bandgap Microcavities in Optical Waveguides", Nature, 390, pp. 143-145, Nov. 1997. 